\title{
The Impact of Live Commerce on Consumers' Purchase Behavior Under the Background of COVID- 19 in China
}

\author{
Mingyu $\mathrm{Fu}^{1, *}$ \\ ${ }^{1}$ Hobart and William Smith Colleges, 3320 Scandling ctr, Geneva, NY, U.S.A, 14456 \\ *Corresponding author. Email:Mingyu_fu@ sina.com
}

\begin{abstract}
E-commerce has existed for a long time, but the outbreak of the COVID-19 ushered in a new opportunity for its development. Live commerce is a significant branch of e-commerce, which mainly realizes businesses through livestreaming marketing. After COVID-19, more and more people watch the livestreaming, it ushered in explosive growth. In the process of livestreaming marketing, Key Opinion Leaders (KOLs) play a very important role. In 2021, $62 \%$ of advertisers use KOL promotion. This paper mainly discussed live commerce under the background of COVID19 in China. Through literature analysis, this paper discussed the impact factors of live commerce on consumers' purchasing behavior and how KOLs affect consumer behavior positively and negatively. In the last part, this article gave some suggestions to help enterprises to recover from COVID-19.
\end{abstract}

Keywords: Live commerce, Livestreaming, Livestreaming marketing, Key Opinion Leader

\section{INTRODUCTION}

The outbreak of COVID-19 has a significant impact on people's lives and the world economy. Because the pandemic has been existing for years, many industries have been profoundly and permanently changed. Among them, e-commerce is a typical industry, which grows rapidly because of the lockdowns and social distance limitation during the pandemic. Live commerce is an important branch of e-commerce. Live commerce is a relatively new and rapidly growing field, which refers to the combination of its streaming media practice and ecommerce revenue strategy[1]. Compared with Western countries, China is in the leading position in the world in terms of platform e-commerce integration, thus supporting the development of their live commerce[1]. The most important channel to realize live commerce is livestreaming marketing. Livestreaming is a broadcast video streaming service provided by Web-based platforms and mobile applications that have synchronous and cross-modal (video, text, and image) interactivity[1]. With the development of mobile Internet in recent years and the impact of COVID-19, livestreaming is the latest form of shopping in China. Consumers can interact with credible audience and valuators in real time, thus obtain a more immersive and informative shopping experience than what brick-and-mortar shops and e-commerce platforms can provide. In 2019, the total value of China's livestreaming e-commerce industry reached 66.8 billion dollars, which was expected to double by the end of 2020 due to the impact of the pandemic of COVID-19[2]. In addition to the audience, the live streamer is also an important part of livestreaming marketing. They realize the mastery of discourse power by constructing a unique persuasion system, so as to provide their fans and the public with a variety of forms of products and content, and through the audience's recognition to achieve sales[3]. The key live streamers with an active influence on consumers can be called the Key Opinion Leaders (KOLs). They play an important role in live commerce. Research of China's marketing trends in 2021 shows that $51 \%$ of advertisers chose to use livestreaming marketing, and $62 \%$ of them use KOL promotion[4]. This paper researches live commerce in China under the background of COVID-19 and finds out the significant role of KOLs in the process. This paper also gives suggestions on how to use livestreaming marketing and KOLs to help recover companies damaged by COVID- 19 . 


\section{LIVE COMMERCE AND CONSUMERS' PURCHASE BEHAVIOR}

Since China's reform and opening up, China's development in science and technology has been very rapid. There is a close relationship between the development of technology and live commerce. The popularity of high-performance mobile phones and the nationwide coverage of network signals play an important role in the development of livestreaming. In addition, China's livestreaming platforms provide integrated e-commerce tools built into the applications to help consumers add products they like to their "shopping cart" at any time while watching videos[5]. This is an important technological basis for the maturity of live commerce in China. The political and economic background of China has promoted the acceptance of live commerce in society. Under the background of China's socialist market economy, a large number of people are enthusiastic about the complete commercialization of personalized social media, which is also the key to China's upgrading from a low-cost manufacturer to a high-value-added technology innovator[1].

The influence factors of live commerce on consumers' purchase behavior are reflected in the following aspects. First, live commerce shortens the time for consumers to make decisions. By blurring the boundaries between social video platforms and ecommerce platforms, live commerce reshapes the decision-making process of customers, making them compress or skip the previous consideration and evaluation process and directly go into the purchase process, thus effectively improving the purchase rate[4]. Second, live commerce attracts consumers and stimulates their purchase decision by providing consumers with credible references. Livestreaming provides a real-time, highly interactive scene. Live streamers can show products comprehensively through video, and interact with the audience in time. All the questions of consumers can be answered in time. Third, live commerce promotes consumers' purchase behavior by creating social pressure. Huseynov and Yildirım found that online shopping pressure from friends, relatives, mass media and other important figures can guide the positive attitude of consumers in any market segment[6]. The livestreaming platforms provide such an environment that viewers can easily be influenced by KOLs, as well as other audiences. Fourth, the intelligent development of content brought about by new technologies can make products more accurate to reach target customers, thus promoting the efficiency of livestreaming marketing. Data platform and content labeling is the most widely used new marketing technology[4]. Through the comprehensive use of new technologies, brands can choose the customers who are most likely to make a purchase decision from a large number of customer groups to carry out marketing.

\section{KOLS IN LIVESTREAMING MARKETING}

Livestreaming seems to be a thing with a low threshold. As long as there is a mobile phone and network signal, anyone can start livestreaming at any time. However, the truly valuable live streamers need to reflect their effective influence on consumers' purchase behavior in livestreaming marketing. Therefore, KOLs are the focus of discussion. Their different behavior may have a positive or negative impact on consumers' behavior. KOLs mainly promote consumers' purchase decisions through A persuasion. Persuasion is a form of utilitarian communication, in which the speaker tries to change the listener's cognition, attitude and behavior[3]. KOLs' persuasion strategies are mainly embodied in specific discourse expressions, speech styles, lifestyle scenes construction and personal traits[3]. Discourse expression is the most basic channel for KOLs to convey information to consumers. Some highly repetitive and special impressive words can attract consumers. For example, Austin Li, one of China's most famous KOLs in the field of livestreaming, often uses phrases such as "Oh My God!", "Buy it! Buy it! Buy it!" to reinforce consumers' purchase behavior. Speech style mainly refers to a KOL's own unique way of emotional expression. Viya, known as the livestreaming marketing queen in China, has her own speech style. She always shows exaggerated and passionate emotional expression in the process of livestreaming, but at the same time gives the audience the most considerate advice when needed. Two completely opposite emotional attitudes shape the unique personality charm of Viya. The figurative means in the process of livestreaming, the scenes behind, the presentation and the construction of lifestyle related to the products are also important means for KOLs to achieve persuasion[3]. It is difficult to use simple descriptive language to convince consumers. Therefore, KOLs need to use figurative means to show the specific features of products more vividly. Scene building is a key piece of information. For example, when beauty KOLs sell cosmetics, it is easier for them to build a lifestyle scene closer to persuade female consumers. Credible similes and scenes can make consumers trust the KOLs and therefore identify with the product. Then, KOLs' personal traits play an important role in the success of persuasion. Personal traits mainly include professional accomplishment and personal morality[3]. Good professional accomplishment is the key to excellent KOLs, and it is also the basis for them to enhance persuasion. Austin Li has adequate expertise in lipsticks. Viya has a full understanding of cosmetics, and her team always takes efforts to deeply investigate the professional knowledge. However, for a KOL and his team, it is impossible to be professional in all fields, so they may lose customer's trust because of the lack of professional knowledge. In 2019, Austin Li displayed a non-stick pan product, but he did not follow the instructions, which led 
to problems[7]. Austin Li, the KOL who had always been trusted, suffered a collapse of trust as a result. Personal morality is also significant. Simba, a famous KOL in livestreaming marketing industry in China, was fined $\$ 900,000$ yuan in the fake goods scandal[2]. The illegal and immoral behavior of selling fake goods made him lose the trust of consumers and caused huge losses.

Online shopping consumers are not a single market segment. They are composed of different consumer groups, and they have different views on online shopping[6]. Therefore, different consumer groups have their own preferences for KOLs. Female consumers like beauty KOLs. Male consumers are more likely to like KOLs of Internet technology and digital products. Young consumers prefer entertainment KOLs, while older consumers may prefer knowledge sharing KOLs. Based on the preferences of different consumers for KOLs, different products have different choices of KOLs to cooperate with. Companies that sell drinks and snacks are more inclined to cooperate with KOLs of food, entertainment and beauty[4]. Internet technology and digital products companies are more likely to work with celebrities in film and television industry and KOLs of entertainment[4]. By cooperating with appropriate KOLs and using livestreaming marketing properly, enterprises can sell products more efficiently.

\section{SUGGESTIONS FOR COMPANIES TO RECOVER FROM COVID-19}

First, companies can effectively use new marketing technologies. Data platform and content labeling mentioned previously are ways to help enterprises effectively process massive data and accurately place livestreaming advertisements. In addition to them, there are some other technologies that can help enterprises improve efficiency and reduce costs. The combination of $\mathrm{AR} / \mathrm{VR}$ and livestreaming marketing can enhance consumers' awareness and trust in products, thus promoting consumer behavior. Artificial intelligence customer service can help enterprises reduce labor costs and improve the response speed to consumers' questions. AI advertising creative technology can help enterprises reduce the cost of advertising design. Virtual live streamers have also become the possible choice. They are more appealing to younger consumers, and the interaction with them is more interesting[4].

Second, enterprises should pay attention to the significance of the awakening of personal social consciousness and empathy after COVID-19. With the influence of the pandemic, people's ideas of lives has changed. People pay more attention to their families and society and hope to actively help social development through personal strength. Therefore, some public welfare actions can stimulate consumers' recognition of corporate social responsibility[4]. For example, livestreaming marketing of agricultural products to promote the concept of helping farmers can attract more consumers to participate in the purchase behavior.

Third, it is very important to choose the right KOL. In the past, many enterprises have chosen to cooperate with celebrities with a huge fan base. However, nowadays, the reputation of the celebrity is more important than the number of fans. Companies need to improve their brand image by cooperating with celebrities with good conduct and personality charm. With the continuous rise of women's consumption power, some mature female celebrities are more and more popular. They are a good choice for companies that want to recover from COVID19.

Fourth, enterprises should make efforts in consumer segmentation and differentiated marketing. Huseynov and Yildirım divided online consumers into four categories: Shopping Lovers, Direct Purchasers, Suspicious Browsers, and Incompetent Consumers[6]. Different types of consumers have different shopping habits and needs. By improving the design and navigation of online stores, the purchase rate of Shopping Lovers can be improved. For Direct Purchasers, enterprises can provide comprehensive information and good service to gain favor. By ensuring the safety of shopping and providing good after-sales service, Suspicious Browsers will tend to buy products. Incompetent Consumers need considerable intelligent systems to help them complete the purchase process. In addition, the pandemic has stimulated online activity among consumers over the age of 50[4]. Therefore, the marketing for middle-aged and elderly groups is worthy of attention.

\section{CONCLUSION}

Under the influence of the pandemic of COVID-19, more and more people choose to shop online, which has stimulated the development of live commerce. Live commerce has become a mature business system in China. Its development is related to the development of technology and the special political and economic background of China. Live commerce influences consumers' purchase behavior by shortening the decision-making time of consumers, providing consumers with trusted references, creating social pressure, and new technology. A very important part of livestreaming marketing is KOLs. They promote consumers' purchase decisions mainly through persuasion. Finally, this paper provides suggestions for enterprises that want to recover from COVID-19. New marketing technologies, presentation of social responsibility, suitable KOLs, consumer segmentation and differentiated marketing are effective means for companies to reduce costs and increase consumer purchase rates. 


\section{ACKNOWLEDGMENTS}

Throughout the writing of this dissertation, I have received a great deal of support and assistance. First, I would like to thank my teachers, Juanjuan Zhang and Min Han, who guided me in doing these projects. They provided me with invaluable advice and helped me in difficult periods. Their motivation and help contributed tremendously to the successful completion of the project. In addition, I am also grateful to my family members for their careful guidance and suggestions that contributed to the completion of this paper.

\section{REFERENCES}

[1] Cunningham, S., Craig, D., \& Lv, J. (2019). China's livestreaming industry: platforms, politics, and precarity. International Journal of Cultural Studies, 22(6), 719-736. https://doi.org/10.1177/1367877919834942

[2] Qiu, Q. L. (2020, Dec 24). Livestreaming company fined $\$ 900,000$ yuan in fake goods scandal. China Daily.

http://www.chinadaily.com.cn/a/202012/24/WS5fe 482b3a31024ad0ba9e5f4.html

[3] Huan, W. (2021). Research on the "Persuasion and Identification" Path of Livestreaming Marketing in the Post-pandemic Era. Broadcasting Realm (03), 38-42. doi: 10.13994/j.cnki.stj.2021.03.010.

[4] Ying, L. (2021). Trends in Social and Content Marketing and KOL Marketing in 2021. China Advertising (02),58-61. doi: CNKI:SUN: gggg.0.2021-02-011.

[5] Shirata, T. (2017, Jun 2). Social commerce, live streaming, and virtual goods: Chinese e-commerce innovation pulls ahead. Guild Investment Management.

https://www.guildinvestment.com/2017/06/02/soci al-commerce-live-streaming-virtual-goods-chinesee-commerce-innovation-pulls-ahead/

[6] Huseynov, F., \& Özkan Yıldırım, S. (2019). Online Consumer Typologies and Their Shopping Behaviors in B2C E-Commerce Platforms. SAGE Open. https://doi.org/10.1177/2158244019854639

[7] The Paper. (2019, Nov 3). Austin Li talked about nonstick pan event: not according to the instructions of the operation, welcome supervision. Sina. https://news.sina.com.cn/s/2019-11-03/dociicezzrr6964743.shtml 